DOI:10.2478/rrlm-2021-0014

\title{
The possibility of clinical use for the oxidative stress marker in correlation with blood flow parameters in pregnancy-induced hypertension
}

\author{
Dragica Draganovic ${ }^{1 *}$, Branka Cancarevic-Djajic ${ }^{1}$, Dragica Jojic ${ }^{2}$, Vesna \\ Ljubojevic $^{3}$, Jelena Todorovic ${ }^{1}$
}

1. Clinic of Gynecology and Obstetrics, University Clinical Centre Banja Luka, Faculty od Medicine, University of Banja Luka, Bosnia and Herzegovina

2. Clinic of Pediatric, University Clinical Centre, Banja Luka, Bosnia and Herzegovina

3. Center for Biomedical Research, Faculty of Medicine, University of Banja Luka, Bosnia and Herzegovina

\begin{abstract}
Introduction: In pregnancy-induced hypertension (PIH) there is a disorder in placental blood flow which causes intrauterine fetal hypoxia, and oxidative stress has a significant role in this condition. The aims of this research were to analyze the relation of thiobarbituric acid reactive substance (TBARS), as a marker of oxidative stress and absent end-diastolic flow (AEDF), as well as the relation of TBARS and cerebroplacental ratio (CPR). Methods: The research included 200 pregnant women in the gestation period from 28th to 40th, 100 were in a control group and 100 were with PIH. The CPR and TBARS were analyzed in all examined pregnant women. The CPR was calculated by dividing the Doppler indices of the middle cerebral artery by the umbilical artery. Results: Mean value of TBARS in the group with the PIH who had AEDF was in the interval of high values $-43.22 \mu \mathrm{mol} / \mathrm{l}$. The result shows that through the application of Spearman's coefficient, the correlation results in a statistically significant correlation between CPR and TBARS values: $\rho=-0.249, p=0.0001$. Conclusion: PIH has a very high level of oxidative stress, especially in pregnant women with absent end-diastolic flow in the umbilical artery and pathologic CPR. The Spearman's test results in statistical significance and negative correlation, which means that in higher TBARS values, values of CPR are lower and vice versa, which indicates a possibility of clinical application of TBARS.
\end{abstract}

Keywords: pregnancy-induced hypertension, thiobarbituric acid reactive substances, cerebroplacental ratio Received: 19 $9^{\text {th }}$ November 2020; Accepted: 24 ${ }^{\text {th }}$ February 2021; Published: $28^{\text {th }}$ February 2021

\footnotetext{
* Corresponding author: Dragica Draganovic, Clinic of Gynecology and Obstetrics, University Clinical Centre Banja Luka, Faculty od Medicine, University of Banja Luka, Bosnia and Herzegovina. E-mail: dragica.draganovic@med.unibl.org
} 


\section{Introduction}

High blood pressure (HBP) represents one of the most common diseases of humanity nowadays, and pregnant women are not exempt from this disease. It is isolated as a special disease that occurs during pregnancy, while the final recovery is accomplished with the termination of pregnancy. For many years, pregnancy-induced hypertension $(\mathrm{PIH})$ has represented a high-risk pregnancy with an incidence of 6-10\%. The etiology of this disease is still unknown [1]. It is characterized by high blood pressure with systolic blood pressure $(\mathrm{SBP})>140 \mathrm{mmHg}$ and diastolic blood pressure $(\mathrm{DBP})>90 \mathrm{mmHg}$, proteinuria greater than $>0.3 \mathrm{~g} / 24 \mathrm{~h}$, and edema after 20 weeks of pregnancy. Hypertensive disorders during pregnancy are classified into 4 categories. As per the recommendation of the National High Blood Pressure Education Program Working Group on High Blood Pressure in Pregnancy, the categories are: chronic hypertension, preeclampsia-eclampsia, preeclampsia superimposed on chronic hypertension, gestational hypertension (transient hypertension of pregnancy or chronic hypertension identified in the latter half of pregnancy). This terminology is preferred to the older but widely used term PIH as it is more precise $[2,3]$. $\mathrm{PIH}$ is a multisystem disorder that involves several organ systems of the pregnant woman and fetus. Hypoperfusion and ischemia of the placenta in PIH occur due to fails of remodeling of the spiral arteries. The ischemic placenta releases factors that provoke a generalized maternal vascular dysfunction, vasoconstriction, followed by consequent hypovolemia, organ hypoperfusion, and uteroplacental hypoperfusion [4-6]. Preeclampsia, as the most common clinical form of this disease, falls within obstetric diseases, which are today the leading cause of maternal and fetal morbidity and mortality in the world, even in the most developed countries. It is responsible for about $14 \%$ of maternal deaths [7-9]. According to recent literature data, it is assumed that PIH is a state of markedly increased oxidative stress in relation to healthy pregnant women without hypertension and it is characterized by a disorder of placental blood flow that results in intrauterine fetal hypoxia. Oxidative stress appears when an imbalance is present between the generation of free radicals and the antioxidant defense systems in the body. Free radicals can alter biomolecules, including the unsaturated fatty acids; deoxyribonucleic acid, ribonucleic acid. More recently, great attention has been paid to lipid peroxidation that involves the unsaturated fatty acids and generates several harmful products including malondialdehyde (MDA) [10-12]. MDA is the main product of lipid peroxidation and an indicator of oxidative stress. The most common and widely available method for measurement is based on the reaction of malondialdehyde with thiobarbituric acid. Thiobarbituric acid reactive substance (TBARS) is a marker of oxidative stress that quickly binds to malondialdehyde.

The application of color Doppler ultrasound in perinatology enables to accurately assess hemodynamic changes in the circulation of the fetus and directly indicate its acute condition. In fetal circulation, the flow in the aorta, umbilical arteries, and cerebral arteries are most often determined by measuring the resistance index (RI). The knowledge regarding flow disorders through umbilical arteries (the resistance index through the umbilical artery - RI UA) and cerebral arteries (the resistance index through the middle cerebral artery - RI MCA) has a prognostic significance for suggesting possible oxygenic complications of the fetus. All pathological conditions in the placenta (sclerosis, degeneration, obliteration, thrombosis) found in the PIH affect the diastolic flow in the arteries of the umbilical cord, which is reflected in the increase of RI value. The absence of diastolic flow in the umbilical artery, absent end-diastolic flow (AEDF), as well as reverse flow, reversed end-diastolic flow (REDF), indicate a severe disorder in fetal cir- 
culation and severe fetal suffering. If there is a decrease in the concentration of $\mathrm{pO}_{2}$ and if the acidosis occurs, the resistance of RI MCA increases, which leads to a decrease in the end-diastolic flow. Numerous perinatal examinations in the detection of the hypoxic fetus, particularly in high-risk pregnancies such as PIH, show that the best clinical application has a combination of these Doppler parameters that is cerebroplacental ratio (CPR) calculation, which is reduced if any of the blood redistributions in favor of the fetal brain occurs. When CPR values are below 1 there is a two-fold increase in the risk of the intrauterine suffering of the fetus and accuracy of the cord arterial $\mathrm{pH}$ less than 7.10 at birth $[13,14]$. It is very important for the course and prognosis of this disease to determine the most optimal time for termination of pregnancy, as definitive therapeutic measures, both in the interest of the mother and in the interest of the fetus. This research is focused on the correlation of the TBARS, as an oxidative stress marker, with fetoplacental flows used in everyday practice to evaluate the intrauterine fetal condition during pregnancy. In recent literature, there is no analysis of all these parameters together.

\section{Methods}

This research was performed at the Clinic of Gynecology and Obstetrics, University Clinical Centre Banja Luka, Bosnia and Herzegovina. The research included a total of 200 pregnant women in the gestation period from $28^{\text {th }}$ to $40^{\text {th }}$. The Ethics Committee of the University Clinical Centre Banja Luka approved the study and informed consent was obtained from all individual respondents. The research was conducted according to the Declaration of Helsinki.

All pregnancies were single with adequate medical documentation during pregnancy, and ultrasound examination was performed during hospitalization. Pregnant women were divided into two groups: 100 pregnant women with PIH that represent the test group and 100 healthy pregnant women that represent the control group. All pregnant women were checked for blood pressure (BP) regularly. The oral regime of therapy was applied by aldomet and nifedipine, depending on the level of BP, high protein nutrition, half-salted nutrition, rest, and oxygenic therapy. $\mathrm{PIH}$ is defined as systolic blood pressure (SBP) $>140 \mathrm{mmHg}$ and diastolic blood pressure (DBP) $>90 \mathrm{mmHg}$. It is classified as mild (if SBP is between $140-149 \mathrm{mmHg}$ and DBP is between $90-99 \mathrm{mmHg}$ ), moderate (if SBP is between $150-159 \mathrm{mmHg}$ and DBP is between 100-109 $\mathrm{mmHg}$ ), and severe (if SBP is $\geq 160$ and DBP is $\geq 110 \mathrm{mmHg}$ ). The selection of pregnant women that belong to the control group was performed after collection of data for the test group, trying to get gestation age and life age of pregnant women in the control and test group as similar as possible. With all researched pregnant women, both control and test group, ultrasound parameters were tested and these were not older than 7 days before the delivery. Ultrasound examination was performed using the device General Electrics GE Logiq BookXP, with the $3.5 \mathrm{MHz}$ convex sonde. The following ultrasound parameters of feto-placental flows were analyzed: cerebroplacental ratio, absent end-diastolic flow and reversed end-diastolic flow. All surveyed pregnant women of the test and control group were blood tested for values of TBARS at The Institute for Laboratory Diagnostics University Clinical Centre Banja Luka. This spectrophotometric method was based on the concentration of malondialdehyde, a product of lipid peroxidation. TBARS level was defined as the equivalent of malondialdehyde standard, following the producer's recommendation (Oxi Select TBARS Analyse Kit MDA quantification), with the use of the spectrophotometric method at $532 \mathrm{~nm}$. Depending on TBARS level there are three intervals. The first interval represents the low val- 
ue if TBARS level is up to $20 \mu \mathrm{mol} / 1$, the medium value if TBARS level is between 20 and 40 $\mu \mathrm{mol} / 1$, and the interval of high value if TBARS level is over $40 \mu \mathrm{mol} / \mathrm{l}$. Statistical analysis was performed with the use of the licensed version of the SPSS 19 statistical software package. Data were analyzed using descriptive statistical methods. Mann-Whitney's U-test, Chi-square $\left(\chi^{2}\right)$ test, the Fisher's test with the appropriate level of significance $\mathrm{p}<0.05$, was used. The degree of connection between TBARS and flow values, i.e. CPR was defined by Spearman's correlation coefficient.

\section{Results}

According to anthropometric parameters for pregnancy, the research results of the group with $\mathrm{PIH}$ and the control group (healthy pregnancy) were analyzed in detail. The medium value of systolic pressure in pregnant women with $\mathrm{PIH}$ was $160 \mathrm{mmHg}$, and in the control group with healthy women, it was $120 \mathrm{mmHg}$, whereas the medium value of diastolic pressure of pregnant women with PIH was $110 \mathrm{mmHg}$, respectively $70 \mathrm{mmHg}$ in healthy pregnant women. By observing the gestational age at delivery, it was found that the pregnancy of women with PIH was shorter, which was highly statistically significant.

The control and test groups with the acquired TBARS values were compared. The mean TBARS value of pregnant women in the control group was $13.24 \pm 2.74 \mu \mathrm{mol} / \mathrm{l}$, and in the test group (pregnant women with $\mathrm{PIH}$ ), the mean TBARS value was $36.69 \pm 8.29 \mu \mathrm{mol} / 1$, which represents the interval of medium values. There was a highly statistically significant difference between the test and control group in the TBARS value $(\mathrm{p}<0.0001)$.

The CPR of both test and control groups were analyzed and systematized (Table 1). Table 1. shows that with the use of the Mann-Whitney's $\mathrm{U}$ test, a statistically significant difference in parameters between the test group of pregnant women with PIH and the control group was determined, except for parameter RI MCA. It was found that in the group of pregnant women with $\mathrm{PIH}$, higher values of the RI umbilical artery were statistically significantly more frequent and the values of CPR were lower. In all pregnant women, Fisher's test showed that the absence of diastole through the umbilical artery was significantly more common in pregnant women with PIH (Table 2).

In order to take into consideration the possibility of clinical application of TBARS as an oxidative stress marker for pregnancy hypertension, it was compared the obtained TBARS mean val-

Table 2. Overview of US pregnancy parameters AEDF in all pregnant women

\begin{tabular}{lccc}
\hline AEDF & PIH group & Control group & $\mathbf{p}$ \\
\hline Yes & 8 & 0 & \multirow{2}{*}{0.0071} \\
No & 92 & 100 & \\
\hline
\end{tabular}

Absent end-diastolic flow (AEDF), ${ }^{1}$ - Fisher's test

Table 1. Overview of feto-placental flows between the test and control group of pregnant women umbilical artery (UA), middle cerebral artery (MCA), cerebroplacental ratio (CPR), MV - medium value, SD standard deviation, Mann-Whitney-test

\begin{tabular}{lccccccccc}
\hline \multirow{2}{*}{ Parameters } & \multicolumn{4}{c}{ PIH group } & \multicolumn{3}{c}{ Control group } & \multicolumn{1}{c}{ p } \\
\cline { 2 - 11 } & Min & Max & MV & SD & Min & Max & MV & SD & \\
\hline Delivery gestation (days) & 203 & 280 & 261.94 & 20.39 & 229 & 280 & 273.96 & 8.56 & 0.0001 \\
\hline RI UA & 0.51 & 0.85 & 0.63 & 0.08 & 0.51 & 0.66 & 0.59 & 0.03 & 0.001 \\
\hline RI MCA & 0.49 & 0.84 & 0.67 & 0.07 & 0.5 & 0.76 & 0.67 & 0.03 & 0.377 \\
\hline CPR & 0.61 & 1.45 & 1.05 & 0.16 & 0.91 & 1.50 & 1.13 & 0.07 & 0.0001 \\
\hline
\end{tabular}


ues with ultrasound (US) parameters: CPR and AEDF. In correlation to the US parameters with TBARS values, when applying Mann-Whitney's U test, there was no statistically significant difference in TBARS value of pregnant women in the test group with AEDF (Md = 43.2250, $\mathrm{n}=$ 8 ) and pregnant women in the test group with the presence of umbilical arterial end-diastolic flow ( $\mathrm{Md}=36.1174, \mathrm{n}=92), \mathrm{U}=227.500, \mathrm{z}$ $=-1.785, \mathrm{p}=0.074$ with $\mathrm{r}=0.179$, (Table 3). Values $\mathrm{p}=0.074$ are borderline values, but these results also deserve attention for future research. All pregnant women in the control group had a regular flow through the umbilical artery.

CPR, an important parameter for intrauterine fetal condition, was conducted in all pregnant women (Table 4). By applying Spearman's correlation coefficient, a weak correlation between CPR and TBARS value $(r=-0.249, p=0.0001)$ in women with PIH and the control group in healthy pregnant women (Figure 1) was discovered. Pregnant women with PIH had significantly lower values of CPR, and high TBARS values, the oxidative stress marker. The correlation between CPR and TBARS values $(r=-0.249)$ was negative, and showed that in higher TBARS values, CPR values were lower.

\section{Discussion}

Examining possibilities of clinical application of TBARS as an oxidative stress marker, this research was focused on the analysis of TBARS value in test and control groups compared to US parameters for the course of pregnancy. Medium

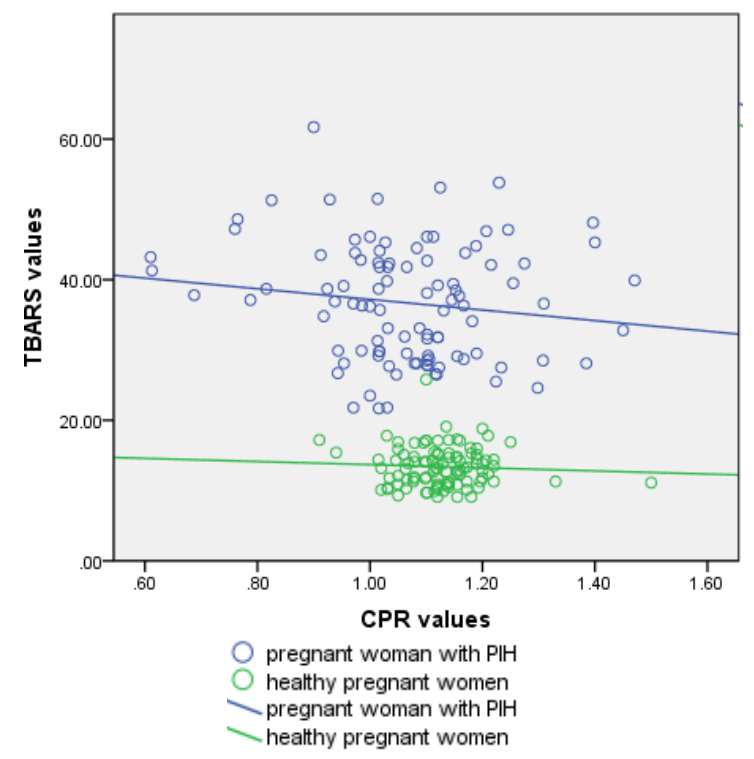

Fig.1. The correlation between TBARS and CPR in pregnant women with PIH and healthy pregnant woomen

Table 3. TBARS values of women with PIH compared to US parameters AEDF

\begin{tabular}{lccc}
\hline AEDF & PIH group & TBARS $(\overline{\mathrm{X}} \pm \mathbf{\text { SD } \boldsymbol { \mu m o l } / \mathbf { l } )}$ & $\mathbf{p}$ \\
\hline Yes & 8 & $43.22 \pm 8.99$ & \\
\hline No & 92 & $36.12 \pm 8.03$ & 0.074 \\
\hline Total & 100 & $36.69 \pm 8.29$ & \\
\hline
\end{tabular}

AEDF - Absent end-diastolic flow in the umbilical artery

Table 4. Analysis of TBARS and CPR values in all pregnant women

\begin{tabular}{lccc}
\hline Parameters & PIH group & Control group & p \\
\hline CPR,$(\overline{\mathrm{X}})$ & 1.049 & 1.127 & 0.0001 \\
\hline TBARS,$(\overline{\mathrm{X}} \pm \mathrm{SD})$ & 36.69 & 13.24 & \\
\hline $\mathrm{N}$ & 100 & 100 & $-0.249^{* *}$ \\
\hline
\end{tabular}

Cerebroplacental ratio (CPR), ${ }^{* *}$ Spearman's correlation coefficient, $r, p<0.01$ 
values of gestational age at delivery were analyzed and proved high statistical significance pregnant women with PIH delivered a baby at earlier gestational age compared to healthy pregnant women [15].

The role of oxidative stress is stated as the cause of many diseases in numerous studies [12,1921]. In this research, medium values of TBARS were analyzed, as the oxidative stress marker, and compared with ultrasound parameters. There was a difference between the test and control group, which confirmed the significance of these parameters for monitoring and evaluation of intrauterine fetal condition in PIH. The obtained mean TBARS values for the control group of pregnant women were within the interval of low values and were $13.24 \mu \mathrm{mol} / \mathrm{l}$. In the test group, TBARS middle values were $36.69 \mu \mathrm{mol} / 1$ and these values were within the interval of medium values. It could be concluded that pregnant women with hypertension had increased oxidative stress. Similar results were obtained in the study by Kumari et al [22]. In this study, the malondialdehyde, which forms a 1:2 adduct with thiobarbituric acid, was found to be elevated in the plasma of women with pre-eclampsia [22]. Measuring the flow in the umbilical artery is a routine, a simple method considering the fact that the umbilical cord is easy to detect and that is available for examination. Findings on the disorder of the flow through the umbilical artery and RI values have prognostic importance which suggests the possible oxygenation complications in the fetus, identifying at-risk fetuses especially in high-risk pregnancies such as PIH. In clinical work, measuring RI flow through MCA is performed simultaneously with measuring RI flow through the umbilical artery. RI is proportional to the concentration of $\mathrm{pO}_{2}$ in fetal blood vessels. In normal conditions of the oxygen concentration, RI MCA is higher than the RI AU. In conditions of severe hypoxia, the RI MCA of the fetus is reduced in order to allow greater blood flow to the brain, ie centralization of blood flow [14-18].

While analyzing AEDF of the tested group and TBARS values from the acquired results, it was noticed that there was no difference in TBARS values between pregnant women with PIH whose ultrasound showed AEDF and those whose ultrasound did not show AEDF. The results deserve attention, considering the obtained values. The mean value of TBARS in a group of pregnant women with PIH who had AEDF was within the interval of high values and it was $43.22 \mu \mathrm{mol} / 1$. Defining TBARS values for pregnant women with PIH that were diagnosed with AEDF on the ultrasound, as in the presented study, is important and could have clinical application.

Through the analysis of the control group of pregnant women, it could be also noticed that they had a regular flow of RI UA, and TBARS medium value was $13.24 \mu \mathrm{mol} / 1$ within the interval of low values. It is significant to emphasize that, while analyzing a control group of pregnant women, 200 of them in total, we did not encounter reversed end-diastolic flow, the US parameter that is always an indicator of acute intrauterine fetus suffering which means that the pregnancy has to be terminated immediately.

In this research, we thoroughly analyzed the US parameter - CPR, that represents the relationship between the resistance index RI flow through the middle cerebral artery and the resistance index RI through the umbilical artery. The CPR lower than 1 , or according to recent literature below 1.08 , reflects centralization of blood circulation and represents a highly sensitive US parameter for the detection of intrauterine fetal hypoxia [23-26]. Obtained results showed that CPR value 1 and lower was significantly more frequent in women with PIH. Therefore, in this research, the correlation of this parameter with TBARS mean values was studied. Pregnant women with lower CPR values had higher TBARS values, hence there was a negative correlation in the ac- 
quired results of these two parameters. TBARS values were analyzed according to intervals of low and medium values and CPR in the control group of pregnant women. Out of 100 pregnant women of the control group, 99 of them had TBARS values within the interval of low values up to $20 \mu \mathrm{mol} / 1$, and a single pregnant woman was within the interval of medium values. CPR for all pregnant women was higher than 1 , which represents a normal finding.

The CPR ratio is of great importance for intrauterine monitoring of the fetal condition, especially for preeclampsia. The correlation between CPR and TBARS value was acquired. According to this test, there was a negative correlation, which meant that in higher TBARS values, values of CPR were lower and vice versa.

There are several studies in the literature, which show that oxidative stress is extremely high in $\mathrm{PIH}$ and that it is in direct positive correlation with values of blood pressure level - with the highest levels of BP, the highest are TBARS values as an oxidative stress marker [10,11,27]. Similar research in the study by Draganovic et al [28] shows that TBARS values, as an oxidative stress marker, are significantly increased in the most severe clinical forms of PIH.

Based on the results obtained in this research, a clear connection of TBARS values and CPR was obtained, as a very sensitive ultrasound parameter for the intrauterine fetal condition, thus it could be concluded that this marker could have a clinical application in the evaluation of intrauterine fetal condition and estimation of the optimal time for pregnancy termination in pregnancies with PIH. However, it requires additional examinations.

The limitation of this study is in the number of the examined pregnant women, and a larger number would significantly improve the possibility of clinical application of TBARS and CPR values. Especially, the clinical application in terms of timely termination of pregnancy in the interest of the fetus should be based on the research of TBARS and CPR values, considering the larger number of pregnant women with severe preeclampsia and separately in pregnant women with mild preeclampsia. Another limitation of the study could be the significant difference in gestational age at delivery between healthy pregnant women and pregnant women with PIH, who had pregnancy termination at an earlier gestational age. The difference in gestational age could affect the difference in oxidative stress between healthy pregnant women and pregnant women with $\mathrm{PIH}$.

\section{Conclusion}

TBARS, as the marker of oxidative stress in pregnant women with PIH is significantly higher compared to TBARS in healthy pregnant women. In pregnant women with PIH, CPR is significantly lower compared to healthy pregnant women. In this study, it is shown that there is a negative correlation between TBARS and CPR values in pregnant women with PIH. Pregnant women with PIH had high values of TBARS, the medium value of $36.69 \mu \mathrm{mol} / 1$, and low values of CPR, the medium value has been 1.05. Whereas healthy pregnant women had lower values of TBARS, medium value of 13.24 $\mu \mathrm{mol} / 1$, and higher values of CPR, the medium value of 1.13. Increased values of TBARS from the blood of pregnant women with PIH could indicate increased oxidative stress, hypoxia, and intrauterine fetal distress. The obtained negative correlation between TBARS, as a marker of oxidative stress, and CPR allows the clinical application of TBARS as part of routine prenatal clinical diagnostic for determining the optimal time for pregnancy termination in the interest of the fetus in pregnant women with PIH.

\section{Abbreviations}

AEDF - absent end-diastolic flow 
BP - blood pressure

CPR - cerebroplacental ratio

DBP - diastolic blood pressure

HBP - high blood pressure

MDA - malondialdehyde

PIH - pregnancy-induced hypertension

REDF - reversed end-diastolic flow

RI - resistance index

RI MCA - resistance index through the middle cerebral artery

RI UA - resistance index through the umbilical artery

SBP - systolic blood pressure

TBARS - thiobarbituric acid reactive substance

US - ultrasound

\section{Authors' contribution}

D.D. - conceptualization, investigation, methodology, writing - original draft

B.Č.Đ. - investigation, writing - review, and editing

D.J. - methodology, data curation, critical revision of the manuscript

V. Lj. - formal analysis, data curation, critical revision of the manuscript

J.T. - investigation, critical revision of the manuscript.

\section{Acknowledgments}

This study was not financially supported.

\section{Conflict of interest}

The authors declare no conflict of interest.

\section{References}

1. Kintiraki E, Papakatsika S, Kotronis G, Goulis DG, Kotsis V. Pregnancy-Induced hypertension. Hormones (Athens) 2015;14(2):211-23. DOI: 10.14310/ horm.2002.1582

2. Program N. H. B. P. E. Report of the National High Blood Pressure Education Program Working Group on High Blood Pressure in Pregnancy. Am J Obstet Gynecol 2000;183(1):S1-S22. DOI: 10.1067/ mob.2000.107928

3. Brown MA, Magee LA, Kenny LC, Karumanchi SA, McCarthy FP, Saito S, et al. Hypertensive disorders of pregnancy: ISSHP classification, diagnosis, and management recommendations for international practice. Hypertension 2018;72(1):24-43. DOI: 10.1161/HYPERTENSIONAHA.117.10803

4. Sava RI, March KL, Pepine CJ. Hypertension in pregnancy: Taking cues from pathophysiology for clinical practice. Clinical cardiology 2018;41(2):220-227. DOI: 10.1002/clc. 22892

5. Lenfant C. National Education Program Working Group on High Blood Pressure in Pregnancy. Working group report on high blood pressure in pregnancy. J Clin Hypertens (Greenwich) 2001;3(2):75-88. DOI: 10.1111/j.1524-6175.2001.00458.x

6. Shah DA, Khalil RA. Bioactive factors in uteroplacental and systemic circulation link placental ischemia to generalized vascular dysfunction in hypertensive pregnancy and preeclampsia. Biochemical pharmacology 2015;95(4):211-226. DOI: 10.1016/j.bcp.2015.04.012

7. Poon LC, Shennan A, Hyett JA, Kapur A, Hadar E, Divakar H, et al. The International Federation of Gynecology and Obstetrics (FIGO) initiative on pre-eclampsia: A pragmatic guide for first-trimester screening and prevention. Int J Gynaecol Obstet 2019;145(Suppl 1):1-33. DOI: $10.1002 /$ ijgo. 12892

8. Hitti J, Sienas L, Walker S, Benedetti TJ, Easterling T. Contribution of hypertension to severe maternal morbidity. Am J Obstet Gynecol 2018;219(4):405.e1-405. e7. DOI: 10.1016/j.ajog.2018.07.002

9. Creanga AA. Maternal mortality in the United States: a review of contemporary data and their limitations. Clin Obstet Gynecol 2018;61(2):296-306. DOI: 10.1097/ GRF.0000000000000362

10. Aouache R, Biquard L, Vaiman D, Miralles F. Oxidative stress in preeclampsia and placental diseases. Int J Mol Sci 2018;19(5):1496-29. DOI: 10.3390/ijms19051496

11. Oztas E, Ozler S, Tokmak A, Erel O, Ergin M, Uygur D, Danisman N. Oxidative stress markers in severe preeclampsia and preeclampsia-related perinatal morbidity - preliminary report. Ginekol Pol 2016;87(6):436-441. DOI: $10.5603 /$ GP.2016.0022

12. Fogarasi E, Croitoru MD, Fülöp I, Nemes-Nagy E, Tripon RG, Simon-Szabo Z, Muntean DL. Malondi- 
aldehyde levels can be measured in serum and saliva by using a fast HPLC method with visible detection/ Determinarea printr-o metodă HPLC-VIS rapidă a concentraţiilor serice şi salivare ale malondialdehidei. Rev Romana Med Lab 2016;24(3):319-326. DOI: 10.1515/ rrlm-2016-0029

13. Bhattacharya SK, Kundu S, Kabiraj SP. Prediction of Preeclampsia by Midtrimester Uterine Artery Doppler Velocimetry in High-Risk and Low-Risk Women. J Obstet Gynaecol India 2012;62(3):297-300. DOI: $10.1007 / \mathrm{s} 13224-012-0219-8$

14. Nagar T, Sharma D, Choudhary M, Khoiwal S, Nagar RP, Pandita A. The Role of Uterine and Umbilical Arterial Doppler in High-risk Pregnancy: A Prospective Observational Study from India. Clin Med Insights Reprod Health 2015;9:1-5. DOI: 10.4137/CMRH.S24048

15. Novac MV, Moldoveanu A, Tudorache Ş, Oprescu ND, Miescu M, Siminel MA, et al. Utility of Cerebroplacental Ratio in IUGR Fetuses from Pregnancy with Preeclampsia in Prediction the Risk for Perinatal Complications. Curr Health Sci J 2017;43(3):231-235.

16. O'neillE, Thorp J.Antepartum evaluation of the fetus and fetal well being. Clin Obstet Gynecol 2012;55(3):722730. DOI: $10.1097 /$ GRF.0b013e318253b318

17. Madazli R, Uludağ S, Ocak V. Doppler assessment of umbilical artery, thoracic aorta and middle cerebral artery in the management of pregnancies with growth restriction. Acta Obstet Gynecol Scand 2001;80(8):702707. DOI: $10.1034 /$ j.1600-0412.2001.080008702.x

18. Sherman RW, Bowie RA, Henfrey MM, Mahajan RP, Bogod D. Cerebral hemodynamics in pregnancy and pre-eclampsia as assessed by transcranial Doppler ultrasonography. Br J Anaesth 2002;89(5):687-692. DOI: 10.1093/bja/89.5.687

19. Ruder EH, Hartman TJ, Blumberg J, Goldman MB. Oxidative stress and antioxidants: exposure and impact on female fertility. Hum Reprod Update 2008;14(4):345357. DOI: 10.1093/humupd/dmn011

20. Scripcariu ȘI, Avasiloaiei A, Socolov D, Mihălceanu E, Dimitriu DC, Moscalu M, Stamatin M. Total antioxidant status as marker of oxidative stress in infants with intrauterine growth restriction. Rev Romana Med Lab
2020;28(2):145-52. DOI: 10.2478/rrlm-2020-0013

21. Gilbert JS, Ryan MJ, LaMarca BB, Sedeek M, Murphy SR, Granger JP. Pathophysiology of hypertension during preeclampsia: linking placental ischemia with endothelial dysfunction. Am J Physiol Heart Circ Physiol 2008;294:H541-550. DOI: 10.1152/ ajpheart.01113.2007

22. Kumari R, Kumar G, Chetri N, Bhattacharya G. Oxidative stress in pre-eclampsia-a study on correlation between malondialdehyde and lipid profile from maternal and cord blood. J Evol Med Dent Sci 2017;6(82):57555759. DOI: $10.14260 /$ jemds/2017/1248

23. Riskin-Mashiah S, Belfort MA, Saade GR, Herd JA. Transcranial Doppler measurement of cerebral velocity indices as a predictor of preeclampsia. Am J Obstet Gynecol 2002;187(6):1667-1672. DOI: 10.1067/ mob.2002.127594

24. Deora R, Gara N. The value of the middle cerebral to umbilical artery doppler ratio in the prediction of neonatal outcome in patients with high risk pregnancy. Int J Reprod Contracept Obstet Gynecol 2017;6(9):39653968. DOI: $10.18203 / 2320-1770$. ijrcog20174045

25. Janas P, Staroń A, Wilczyńska G, Brzozowska M. The role of cerebroplacental ratio in prediction of neonatal outcomes and route of delivery: PS134. Porto Biomed J 2017;2(5):239. DOI: 10.1016/j.pbj.2017.07.148

26. Vergani P, Roncaglia N, Locatelli A, Andreotti C, Crippa I, Pezzullo JC, Ghidini A. Antenatal predictors of neonatal outcome in fetal growth restriction with absent end-diastolic flow in the umbilical artery. Am J Obstet Gynecol 2005;193(3):1213-1218. DOI: $10.1016 / j$. ajog.2005.07.032

27. Lurba E, Gratacós E, Martín-Gallán P, Cabero L, Dominguez C. A comprehensive study of oxidative stress and antioxidant status in preeclampsia and normal pregnancy. Free Radic Biol Med 2004;37(4):557570. DOI: 10.1016/j.freeradbiomed.2004.04.035

28. Draganović D, Lučić N, Jojić D. Oxidative Stress Marker and Pregnancy Induced Hypertension. Med Archiv 2016;70(60):437-441. DOI: 10.5455/medarh.2016.70.437-440 